\title{
Symmetry Breaking in Heisenberg Antiferromagnets
}

\author{
Tohru Koma, Hal Tasaki \\ Department of Physics, Gakushuin University, Mejiro, Toshima-ku, Tokyo 171, Japan
}

Received: 17 November 1992/in revised form: 16 February 1993

\begin{abstract}
We extend Griffith's theorem on symmetry breaking in quantum spin systems to the situation where the order operator and the Hamiltonian do not commute with each other. The theorem establishes that the existence of a long range order in a symmetric (non-pure) infinite-volume state implies the existence of a symmetry breaking in the state obtained by applying an infinitesimal symmetry-breaking field. The theorem is most meaningful when applied to a class of quantum antiferromagnets where the existence of a long range order has been proved by the Dyson-Lieb-Simon method. We also present a related theorem for the ground states. It is an improvement of the theorem by Kaplan, Horsch and von der Linden. Our lower bounds on the spontaneous staggered magnetization in terms of the long range order parameter take into account the symmetry of the system properly, and are likely to be saturated in general models.
\end{abstract}

\section{Introduction}

Among the most important issues in rigorous statistical mechanics is to establish the existence of phase transitions in various idealized models of physical systems. When a transition is accompanied by a breakdown of a discrete symmetry, the Peierls' argument and its variants [1] can be applied to produce strong rigorous results. When a transition is accompanied by a breakdown of a continuous symmetry, on the other hand, the Peierl's method does not work in general. Fröhlich, Simon and Spencer [2] developed a method based on reflection positivity [3], which enabled them to prove the existence of phase transitions in various classical spin systems with continuous symmetry. The method was extended to quantum spin systems by Dyson, Lieb and Simon [4]. Their result is outstanding in that it rigorously establishes the existence of phase transitions in physically realistic models, such as the three-dimensional quantum Heisenberg antiferromagnets.

In order to motivate the theorem of the present paper, we shall describe the main result of Dyson, Lieb and Simon in the context of Heisenberg antiferromagnets. Take 
a $d$-dimensional $L \times \cdots \times L$ hypercubic lattice $\Lambda$ with periodic boundary conditions, where $L$ is an even integer. With each site $x=\left(x_{1}, \ldots, x_{d}\right) \in \Lambda$, we associate three component quantum mechanical spin operators $\mathbf{S}_{x}=\left(S_{x}^{(1)}, S_{x}^{(2)}, S_{x}^{(3)}\right)$ with $\left(\mathbf{S}_{x}\right)^{2}=S(S+1)$, where $S=1 / 2,1,3 / 2, \ldots$. The Hamiltonian for the isotropic Heisenberg antiferromagnet is

$$
H_{\Lambda}:=\sum_{x, y \in \Lambda:|x-y|=1} \mathbf{S}_{x} \cdot \mathbf{S}_{y} .
$$

Throughout the present paper, the symbol := signifies a definition.

Dyson, Lieb and Simon [4] proved that the long range order parameter $\sigma$ satisfies

$$
\sigma:=\lim _{L \rightarrow \infty} \frac{1}{L^{d}} \sqrt{\left\langle\left(O_{\Lambda}^{(1)}\right)^{2}\right\rangle_{\Lambda}}>0
$$

at sufficiently low temperature if $S \geq 1$ in $d \geq 3$. Here the order operators are

$$
O_{\Lambda}^{(i)}:=\sum_{x \in \Lambda}(-1)^{x_{1}+\cdots+x_{d}} S_{x}^{(i)}
$$

for $i=1,2,3$, and

$$
\langle\cdots\rangle_{\Lambda}:=\frac{\operatorname{Tr}\left[\cdots e^{-\beta H_{\Lambda}}\right]}{\operatorname{Tr}\left[e^{-\beta H_{\Lambda}}\right]},
$$

with the inverse temperature $\beta$. The relation (1.2) establishes that the system develops a Néel-type long range order at sufficiently low temperatures. By using the improvement due to Kennedy, Lieb and Shastry [5], this result was extended [6] to the model in $d=3$ with $S=1 / 2$. (There are also various extensions to ground states [7], to anisotropic models [6,8], and to models with certain frustrations [9].)

To investigate physical consequences of the result of Dyson, Lieb and Simon, consider the infinite volume equilibrium state $\omega_{0}(\cdots)$ constructed as

$$
\omega_{0}(\cdots):=\lim _{L \rightarrow \infty}\langle\cdots\rangle_{\Lambda} .
$$

Since $\omega_{0}(\cdots)$ inherits the $S U(2)$ invariance of the Hamiltonian $H_{\Lambda}$, we see that it does not break the $S U(2)$ symmetry. In particular, we observe that

$$
\omega_{0}\left(\mathbf{S}_{x}\right)=\mathbf{0}
$$

The relation (1.2), on the other hand, implies that

$$
\omega_{0}\left(\mathbf{S}_{x} \cdot \mathbf{S}_{y}\right) \not 00 \text { as }|x-y| \rightarrow \infty
$$

The relations (1.6) and (1.7) indicate that the truncated correlation function $\omega_{0}\left(\mathbf{S}_{x} \cdot \mathbf{S}_{y}\right)-\omega_{0}\left(\mathbf{S}_{x}\right) \cdot \omega_{0}\left(\mathbf{S}_{y}\right)$ does not decay to zero at large distances, and hence $\omega_{0}(\cdots)$ is not a pure state. It is believed that a physically realizable equilibrium state in a sufficiently large system is well approximated by a pure state. From a physical point of view, a non-pure state may be regarded as an "unnatural" state. One must be careful about what is the physical implication of the relation (1.2). (But see Remarks 1 and 2 at the end of the present section.) 
In physics literature, a standard way to get an infinite volume state with explicit symmetry breaking is to apply an infinitesimally small symmetry breaking field to the system. In the case of the Heisenberg antiferromagnet, this can be implemented as

$$
\tilde{\omega}(\cdots)=\lim _{B \downarrow 0} \lim _{L \uparrow \infty} \frac{\operatorname{Tr}\left[\cdots \exp \left[-\beta\left(H_{\Lambda}-B O_{\Lambda}^{(1)}\right)\right]\right]}{\operatorname{Tr}\left[\exp \left[-\beta\left(H_{\Lambda}-B O_{\Lambda}^{(1)}\right)\right]\right]} .
$$

It is likely that the state $\tilde{\omega}(\cdots)$ is pure, but there is no proof. It is natural to expect that the existence of a long range order in the $S U(2)$ invariant state $\omega_{0}(\cdots)$ [as in (1.2)] implies a symmetry breaking in the state $\tilde{\omega}(\cdots)$. The latter is usually characterized by observing that the spontaneous staggered magnetization

$$
\begin{aligned}
m_{\mathrm{s}} & :=\lim _{B \downarrow 0} \lim _{L \uparrow \infty} \frac{1}{L^{d}} \frac{\operatorname{Tr}\left[O_{\Lambda}^{(1)} \exp \left[-\beta\left(H_{\Lambda}-B O_{\Lambda}^{(1)}\right)\right]\right]}{\operatorname{Tr}\left[\exp \left[-\beta\left(H_{\Lambda}-B O_{\Lambda}^{(1)}\right)\right]\right]} \\
& =(-1)^{x_{1}+\cdots+x_{d}} \tilde{\omega}\left(S_{x}^{(1)}\right)
\end{aligned}
$$

takes a nonvanishing value. (The equality in (1.9) follows from the translation invariance.) Mathematically speaking, however, the implication of nonvanishing $\sigma$ on nonvanishing $m_{\mathrm{s}}$ is not as trivial as it appears.

The method of Dyson, Lieb and Simon can deal only with the $S U(2)$ invariant state $\omega_{0}(\cdots)$, and not with the state $\tilde{\omega}(\cdots)$ obtained by applying an infinitesimal symmetry breaking field. In models where the order operators and the Hamiltonian commute with each other (such as the Heisenberg ferromagnets), Griffiths [10] developed a general theory which relates a symmetry breaking in states like $\tilde{\omega}(\cdots)$ with a long range order in the symmetric states like $\omega_{0}(\cdots)$. This result was reformulated and refined by Dyson, Lieb and Simon [4]. Unfortunately, the reflection-positivity method to prove a long range order works only in the antiferromagnetic models, where the order operators and the Hamiltonian do not commute with each other. Therefore the existence of a symmetry breaking in the state $\tilde{\omega}(\cdots)$ in the Heisenberg antiferromagnet has been left as an open problem.

The main purpose of the present paper is to prove a theorem which fills this gap. More precisely, we extend Griffith's theorem mentioned above to models where the order operator and the Hamiltonian do not commute with each other. When applied to the Heisenberg antiferromagnet, Corollary 2.2 in Sect. 2 reads as the following.

Corollary 1.1. In the isotropic Heisenberg antiferromagnet (1.1), the long range order parameter (1.2) and the spontaneous staggered magnetization (1.9) satisfy

$$
m_{\mathrm{s}} \geq \sqrt{3} \sigma
$$

for any inverse temperature $\beta$.

When the Dyson-Lieb-Simon method works, one knows that $\sigma$ is nonvanishing. When combined with such information, our theorem concludes that the state $\tilde{\omega}(\cdots)$ exhibits the spontaneous symmetry breaking in the sense that $m_{\mathrm{s}}>0$. We note that the first rigorous result concerning the existence of such a symmetry breaking in the Heisenberg antiferromagnet was due to Kaplan, Horsch and von der Linden, who proved the bound $m_{\mathrm{s}} \geq \sigma$ for the ground state [11]. We believe that the above bound (1.10) is saturated in the Heisenberg antiferromagnet. See Remarks 3 and 4 below for discussions on the factor $\sqrt{3}$. When the system has a different symmetry group 
than $S U(2)$, the factor is replaced with a proper one. See the Remark at the end of Sect. 6.

Since the bound (1.10) is valid for any temperature, it applies to the ground state obtained by letting $\beta \rightarrow \infty$ after the infinite volume limit. (This is the physically natural ground state.) We also directly treat the ground states in a finite volume, and prove a bound corresponding to (1.10). It is an improvement of a bound by Kaplan, Horsch and von der Linden [11].

Underlying most of our proofs in the present paper is the fact that, in a large quantum system, intensive quantities should behave almost as "classical" quantities. We shall make this claim rigorous in various different ways to prove our results. One of the most interesting techniques in the present work is the "coarse graining" procedure used in Sect. 3 to get a pair of commuting operators from non-commuting ones. This allows us to apply the original idea of Griffiths [10] in order to get the desired relation between long range order and symmetry breaking.

The organization of the present paper is as follows. In Sect. 2, we describe our theorems for finite temperatures in the most general setting. In Sect. 3, we construct a pair of commuting operators by using a kind of coarse graining procedure in the energy space. In Sect. 4, we generalize Griffith's argument, and discuss the probability distribution of the approximate magnetization. We complete the proof of the main theorem for an equilibrium state in Sect. 5. In Sect. 6, we prove general results which characterize a state with continuous symmetry. Section 7 is an independent section which is devoted to the study of ground states.

Remarks. 1. Abstract theories of quantum mechanical states [12] tell us that a nonpure state is always decomposed into a sum of pure states. In the Heisenberg antiferromagnet, it is likely (but not proved) that the state $\omega_{0}(\cdots)$ of (1.5) is decomposed as

$$
\omega_{0}(\cdots)=\frac{1}{4 \pi} \int d \boldsymbol{\Omega} \omega_{\boldsymbol{\Omega}}(\ldots)
$$

where $\boldsymbol{\Omega}$ is the solid angle, and the integration is over the whole sphere. Each state $\omega_{\Omega}(\cdots)$ is pure and exhibits explicit symmetry breaking as

$$
\omega_{\mathbf{\Omega}}\left(\mathbf{S}_{x}\right)=(-1)^{x_{1}+\cdots+x_{d}} m_{\mathrm{s}} \mathbf{\Omega}
$$

with the spontaneous staggered magnetization $m_{\mathrm{s}}$.

2. Given the fact that there is a non-pure state $\omega_{0}(\cdots)$ with a long range order, general arguments guarantee that there is a pure state with explicit symmetry breaking. Therefore one should not understand our contribution as the "first demonstration that there is symmetry breaking." The most important point of our work is that we have established the property of the specific state $\tilde{\omega}(\cdots)$, which is considered as a physically "natural" state to look at.

3. One should not be surprised by the factor $\sqrt{3}$ in the bound (1.10). Once we assume the pure state decomposition (1.11), we immediately see that (1.10) should be satisfied as an equality. By using the translation invariance, one finds that

$$
\sigma^{2}=\lim _{|x| \uparrow \infty}(-1)^{x_{1}+\cdots+x_{d}} \omega_{0}\left(S_{0}^{(1)} S_{x}^{(1)}\right)=\frac{1}{3} \lim _{|x| \uparrow \infty}(-1)^{x_{1}+\cdots+x_{d}} \omega_{0}\left(\mathbf{S}_{0} \cdot \mathbf{S}_{x}\right)
$$


where $\mathbf{S}_{0}$ denotes the spin at the origin, and we have used the $S U(2)$ invariance. By using the decomposition (1.11) and (1.12), we find that

$$
\begin{aligned}
& (-1)^{x_{1}+\cdots+x} d \omega_{0}\left(\mathbf{S}_{0} \cdot \mathbf{S}_{x}\right)=\frac{1}{4 \pi} \int d \mathbf{\Omega}(-1)^{x_{1}+\cdots+x} d \omega_{\mathbf{\Omega}}\left(\mathbf{S}_{0} \cdot \mathbf{S}_{x}\right) \\
& \rightarrow \frac{1}{4 \pi} \int d \boldsymbol{\Omega} \omega_{\boldsymbol{\Omega}}\left(\mathbf{S}_{0}\right) \cdot(-1)^{x_{1}+\cdots+x_{d}} \omega_{\boldsymbol{\Omega}}\left(\mathbf{S}_{x}\right) \\
& =\left(m_{\mathrm{s}}\right)^{2} \text {, }
\end{aligned}
$$

as $|x| \rightarrow \infty$, where we have used that $\omega_{\boldsymbol{\Omega}}$ is pure. This explains (but does not prove!) that we should have $m_{\mathrm{s}}=\sqrt{3} \sigma$ in a "natural" system. Although some authors discussed [11] the possibility of $m_{\mathrm{s}}=r \sigma$ with $r \neq \sqrt{3}$, the above discussion shows that a state must violate the pure state decomposition (1.11) in order to get such a situation, which is rather unlikely. To assume (pure) states with "large quantum fluctuation" is certainly not enough to get $r \neq \sqrt{3}$.

4. Is is easy to construct examples which violate the $r=\sqrt{3}$ rule. Take two independent identical Heisenberg antiferromagnets, and define $O_{\Lambda}^{(1)}$ as the sum of corresponding order operators of each model. Then one immediately finds that the corresponding spontaneous staggered magnetization and the long range order parameter satisfy $m_{\mathrm{s}} \geq \sqrt{6} \sigma$. This observation implies that one cannot prove the equality corresponding to (1.10) without making detailed assumptions on the system.

\section{Notation and Main Results}

In the present section, we shall describe our theorems for finite temperature equilibrium states. The results for ground states are discussed separately in Sect. 7. Although theorems would be most interesting when applied to a class of Heisenberg antiferromagnets where the existence of long range order is known, we shall describe the theorems in their most general forms. We stress that the theorems can deal with a large class of models and various types of long range order. An interesting example is the electron pair condensation problem (superconductivity) in lattice electron systems, but there are no rigorous results on long range orders in fermion systems so far.

We consider a quantum system on a finite lattice $\Lambda \subset \mathbf{Z}^{d}$ with $N$ sites. With each site $x \in \Lambda$, we associate a finite-dimensional Hilbert space $\mathbf{H}_{x}$. The full Hilbert space of the model is

$$
\mathbf{H}_{\Lambda}:=\bigotimes_{x \in \Lambda} \mathbf{H}_{x}
$$

For a fixed $\Lambda$, we take the Hamiltonian

$$
H_{\Lambda}:=\sum_{x \in \Lambda} h_{x}
$$

with self-adjoint operators $h_{x}$. Assume that there is a unitary operator $U_{\Lambda}$ such that

$$
U_{\Lambda} H_{\Lambda} U_{\Lambda}^{*}=H_{\Lambda}
$$

We introduce an "order operator"

$$
O_{\Lambda}:=\sum_{x \in \Lambda} o_{x}
$$


with self-adjoint operators $o_{x}$ to measure a possible spontaneous breakdown of the symmetry generated by the above $U_{\Lambda}$. Thus we require

$$
U_{\Lambda} O_{\Lambda} U_{\Lambda}^{*}=-O_{\Lambda}
$$

We define the Hamiltonian for the system under a "magnetic field" $B \in \mathbf{R}$ (or a "staggered magnetic field" in the case of antiferromagnet) as

$$
H_{\Lambda}(B):=H_{\Lambda}-B O_{\Lambda},
$$

and the corresponding partition function and free energy as

$$
f_{\Lambda}(B):=-\frac{1}{\beta N} \log Z_{\Lambda}(B)
$$

and

$$
Z_{\Lambda}(B):=\operatorname{Tr} e^{-\beta H_{\Lambda}(B)},
$$

respectively. Here $\operatorname{Tr}$ denotes the trace on $\mathbf{H}_{\Lambda}$ and $\beta \geq 0$ is the inverse temperature. As usual we define the thermal expectation as

$$
\langle\cdots\rangle_{\Lambda}(B):=\frac{1}{Z_{\Lambda}(B)} \operatorname{Tr}\left[\cdots e^{-\beta H_{\Lambda}(B)}\right] .
$$

We consider a sequence of finite lattices $\Lambda \subset \mathbf{Z}^{d}$ which tends to the infinite $d$ dimensional hypercubic lattice $\mathbf{Z}^{d}$ in the sense of Van Hove [13]. For each $\Lambda$, we consider a quantum system on $\Lambda$ with the Hamiltonian $H_{\Lambda}$ and the order operator $O_{\Lambda}$. We assume that the sequence of models satisfies the following. (Note that we do not assume that $h_{x}$ and $o_{x}$ are common for different $\Lambda$. This allows one to include various boundary conditions for finite systems.)

i) There exists a thermodynamic limit of the free energy

$$
f(B):=\lim _{\Lambda \uparrow \mathbf{Z}^{d}} f_{\Lambda}(B),
$$

for fixed values of $\beta \geq 0, B \in \mathbf{R}$.

ii) There are finite constants $h$ and $o$ which are independent of $\Lambda$. For each $\Lambda$, we have $\left\|h_{x}\right\|<h$ and $\left\|o_{x}\right\| \leq o$ for any $x \in \Lambda$.

iii) $\left[h_{x}, o_{y}\right]=0$ holds unless $y \in S(x)$. The number of sites in the support set $S(x) \subset \Lambda$ is bounded from above by a constant $r \geq 2$ which is independent of $\Lambda$.

We define the spontaneous (staggered) magnetization as

$$
m_{\mathrm{s}}:=\lim _{B \downarrow 0} \lim _{\Lambda \uparrow \mathbf{Z}^{d}} \frac{1}{N}\left\langle O_{\Lambda}\right\rangle_{\Lambda}(B)
$$

The existence of the limit is guaranteed by the concavity [14] of the free energy $f(B)$ in (2.10). We also define the long range order parameter as

$$
\sigma:=\lim _{\Lambda \uparrow \mathbf{Z}^{d}} \frac{1}{N} \sqrt{\left\langle\left(O_{\Lambda}\right)^{2}\right\rangle_{\Lambda}(0)}
$$

The limit may not exist in general, but one can always take a subsequence so that it exists. 
Then our main result is the following.

Theorem 2.1. For an arbitrary sequence of models satisfying the assumptions i), ii) and iii), we have the inequality

$$
m_{\mathrm{s}} \geq \lim _{\Lambda \uparrow \mathbf{Z}^{d}} \frac{1}{N}\left\{\left\langle\left(O_{\Lambda}\right)^{2 k}\right\rangle_{\Lambda}(0)\right\}^{\frac{1}{2 k}} \geq \sigma
$$

for any inverse temperature $\beta$ and for any positive integer $k$.

Note that the above theorem is a strict extension of Griffith's theorem [10] or Theorem 1.2 of Dyson, Lieb and Simon [4], which are proved assuming that the order operator $O_{\Lambda}$ and the Hamiltonian $H_{\Lambda}$ commute with each other.

The first bound in (2.13) will be proved in Sects. 3, 4 and 5. The second bound is an immediate consequence of the Hölder inequality.

When the system has higher symmetry (as in the Heisenberg antiferromagnets), we can prove bounds stronger than $m_{\mathrm{s}} \geq \sigma$. Although we can treat an almost arbitrary continuous symmetry group, we focus on the most typical case of an $S U(2)$ symmetry.

We now suppose that the order operators form a three component vector $\left(O_{\Lambda}^{(1)}, O_{\Lambda}^{(2)}, O_{\Lambda}^{(3)}\right)$ with

$$
O_{\Lambda}^{(i)}=\sum_{x \in \Lambda} o_{x}^{(\imath)},
$$

where each $o_{x}^{(\imath)}$ is self-adjoint, and the above ii) and iii) are valid for each $i=1,2$ or 3 . We also assume that iv) $\left[o_{x}^{(i)}, o_{y}^{(j)}\right]=0$ for any $x \neq y \in \Lambda$ and any $i, j=1,2,3$.

Finally we require the system to have global $S U(2)$ invariance in the following sense. There are $s u(2)$ generators $X_{\Lambda}^{(1)}, X_{\Lambda}^{(2)}, X_{\Lambda}^{(3)}$, which satisfy the commutation relations

$$
\left[X_{\Lambda}^{(j)}, X_{\Lambda}^{(k)}\right]=i \sum_{\ell=1,2,3} \varepsilon_{\ell}^{j k} X_{\Lambda}^{(\ell)}
$$

where $\varepsilon^{12}{ }_{3}=\varepsilon^{23}{ }_{1}=\varepsilon^{31}{ }_{2}=-\varepsilon^{32}{ }_{1}=-\varepsilon^{21}{ }_{3}=-\varepsilon^{13}{ }_{2}=1$ and $\varepsilon^{\jmath k}{ }_{\ell}=0$ for other $j, k, \ell$.

v) Under the $S U(2)$ transformation, the order operators behave as the tree components of a vector. In other words, the commutation relations

$$
\left[X_{\Lambda}^{(j)}, O_{\Lambda}^{(k)}\right]=i \sum_{\ell=1,2,3} \varepsilon^{j k} O_{\Lambda}^{(\ell)}
$$

hold for any $j, k=1,2,3$.

vi) The Hamiltonian is invariant under the $S U(2)$ transformation. In terms of the commutation relations,

$$
\left[H_{\Lambda}, X_{\Lambda}^{(\jmath)}\right]=0
$$

holds for any $j=1,2,3$.

Then the improved result is the following.

Corollary 2.2. For an arbitrary sequence of models satisfying the assumptions i) vi), we have the inequality

$$
m_{\mathrm{s}} \geq \sqrt{3} \sigma
$$

for any inverse temperature $\beta$. 
Note that this is an extension of Theorem 1.3 of Dyson, Lieb and Simon [4]. The above Corollary follows from Theorem 2.1 and Theorem 6.1.

\section{Construction of Commuting Operators}

This is the first of the succeeding three sections devoted to the proof of Theorem 2.1. In the present section, we will construct a pair of commuting operators $\widetilde{H}_{\Lambda}$ and $\widetilde{O}_{\Lambda}$ which approximate the Hamiltonian $H_{\Lambda}$ and the order operator $O_{\Lambda}$, respectively. As will be seen in Sect. 4, use of these commuting operators is essential in generalizing the technique developed by Griffiths [10]. The construction of commuting operators can be regarded as a kind of "coarse graining" procedure in the energy space. An important feature of the present construction is that the precision of the coarse graining becomes finer and finer as the system size increases (see Proposition 3.2 below).

Throughout the proof in Sects. 3, 4 and 5, the inverse temperature $\beta$ is fixed. In the present section, we also fix a finite lattice $\Lambda$ with $N$ sites, and consider a system with vanishing magnetic field $B$. (To make the following proof simpler, we assume that $N=(4 K)^{4}$, where $K$ is a positive integer. But this technical assumption is by no means essential.) Thus the expectation $\langle\cdots\rangle_{\Lambda}$ always stand for $\langle\cdots\rangle_{\Lambda}(B=0)$. We denote by $\left\{\Phi_{n}\right\}_{n}$ the orthonormal basis formed by eigenstates of $H_{\Lambda}$. The eigenvalue corresponding to the state $\Phi_{n}$ is denoted as $E_{n}$.

Note that any eigenvalue of $H_{\Lambda}$ is contained in the interval

$$
I=[-h N, h N),
$$

from the definition of the Hamiltonian (2.2) and the assumption ii) in Sect. 2. For an arbitrary subset $J \subset I$, we denote by $P(J)$ the projection operator defined by

$$
P(J)=\sum_{n: E_{n} \in J} P_{n}
$$

where $P_{n}$ denotes the orthogonal projection onto the eigenstate $\Phi_{n}$.

Before defining the commuting operators, we have to state one lemma. Let the decomposition number be

$$
M=4 N^{1 / 2}+1
$$

For $\ell=2,3, \cdots, M$ we set

$$
J_{\ell}:=\left[-h N+(\ell-2) \frac{h N^{1 / 2}}{2},-h N+(\ell-1) \frac{h N^{1 / 2}}{2}\right) .
$$

Lemma 3.1. One can find a set $\left\{\widetilde{E}_{\ell}\right\}$ with $\ell=2,3, \ldots, M$ such that $\widetilde{E}_{\ell} \in J_{\ell}$,

$$
\Delta J_{\ell}:=\left[\widetilde{E}_{\ell}-h N^{1 / 4}, \widetilde{E}_{\ell}+h N^{1 / 4}\right) \subset J_{\ell}
$$

and

$$
\sum_{\ell=2}^{M}\left\langle P\left(\Delta J_{\ell}\right)\right\rangle_{\Lambda} \leq 4 N^{-1 / 4}
$$


Proof. We divide each interval $J_{\ell}$ into identical small intervals of length $2 h N^{1 / 4}$ as $J_{\ell}=\Delta J_{\ell}^{(1)} \cup \ldots \cup \Delta J_{\ell}^{(K)}$ with $K=N^{1 / 4} / 4$. Now assume the converse of the desired bond (3.6). Then we must have

$$
\sum_{\ell=2}^{M} \min _{k=1, \ldots, K}\left\langle P\left(\Delta J_{\ell}^{(k)}\right)\right\rangle_{\Lambda}>4 N^{-1 / 4} .
$$

On the other hand, the left-hand side of (3.7) is bounded as

$$
\sum_{\ell=2}^{M} \min _{k=1, \ldots, K}\left\langle P\left(\Delta J_{\ell}^{(k)}\right)\right\rangle_{\Lambda} \leq \sum_{\ell=2}^{M} \frac{1}{K} \sum_{k=1}^{K}\left\langle P\left(\Delta J_{\ell}^{(k)}\right)\right\rangle_{\Lambda}=\frac{1}{K}=4 N^{-1 / 4}
$$

which is a contradiction.

Take and fix $\widetilde{E}_{2}, \ldots, \widetilde{E}_{M}$ as those guaranteed by Lemma 3.1. We also set $\widetilde{E}_{1}=-h N$ and $\widetilde{E}_{M+1}=h N$. We decompose the interval $I$ as

$$
I=I_{1} \cup I_{2} \cup \ldots \cup I_{M}
$$

where $I_{\ell}=\left[\widetilde{E}_{\ell}, \widetilde{E}_{\ell+1}\right)$ for $\ell=1, \ldots, M$. Note that the length of each interval $I_{\ell}$ does not exceed $h N^{1 / 2}$.

We define new operators as

$$
\widetilde{H}_{\Lambda}:=\sum_{\ell=1}^{M} \widetilde{E}_{\ell} P\left(I_{\ell}\right)
$$

and

$$
\widetilde{O}_{\Lambda}:=\sum_{\ell=1}^{M} P\left(I_{\ell}\right) O_{\Lambda} P\left(I_{\ell}\right)
$$

The main result of the present section is the following.

Proposition 3.2. The operators $\widetilde{H}_{\Lambda}$ and $\widetilde{O}_{\Lambda}$ satisfy the following:

$$
\begin{aligned}
{\left[\widetilde{H}_{\Lambda}, \widetilde{O}_{\Lambda}\right] } & =0, \\
\widetilde{H}_{\Lambda} & \leq H_{\Lambda}, \\
\frac{\left\|\widetilde{H}_{\Lambda}-H_{\Lambda}\right\|}{h N} & \leq N^{-1 / 2} .
\end{aligned}
$$

For any eigenstate $\Psi$ of $\widetilde{H}_{\Lambda}$,

$$
\left(\Psi, \widetilde{O}_{\Lambda} \Psi\right)=\left(\Psi, O_{\Lambda} \Psi\right)
$$

For any positive integer $k$

$$
\frac{\left|\left\langle\left(O_{\Lambda}\right)^{2 k}\right\rangle_{\Lambda}-\left\langle\left(\widetilde{O}_{\Lambda}\right)^{2 k}\right\rangle_{\Lambda}\right|}{(o N)^{2 k}} \leq 2 r k 4^{k} N^{-1 / 4}
$$

Before proving the proposition, we state the following lemma. 
Lemma 3.3. For a fixed eigenstate index $n$, take an arbitrary $J \subset I$ such that any $E \in J$ satisfies $\left|E-E_{n}\right| \geq D>0$. Then we have

$$
\left|\left(\Phi_{n}, O_{\Lambda}\left[\prod_{j=1}^{m-1}\left(P_{j} O_{\Lambda}\right)\right] P(J) O_{\Lambda}\left[\prod_{j=m+1}^{2 k-1}\left(P_{j} O_{\Lambda}\right)\right] \Phi_{n}\right)\right| \leq \frac{4 r k h(o N)^{2 k}}{D}
$$

for arbitrary integers $m<2 k$ and arbitrary projection operators $P_{1}, \ldots, P_{m-1}, P_{m+1}$, $\ldots, P_{2 k-1}$ which commute with the Hamiltonian $H_{\Lambda}$.

This lemma implies that the above matrix elements can be made small by choosing sufficiently large $D$. We shall use the lemma with $D=O\left(N^{1 / 4}\right)$ in the proof of Proposition 3.2 .

Proof. Let us write

$$
A^{*}:=O_{\Lambda} \prod_{j=1}^{m-1}\left(P_{\jmath} O_{\Lambda}\right)
$$

and

$$
B:=O_{\Lambda} \prod_{j=m+1}^{2 k-1}\left(P_{j} O_{\Lambda}\right)
$$

By using the Schwarz inequality we get

$$
\begin{aligned}
\left|\left(\Phi_{n}, A^{*} P(J) B \Phi_{n}\right)\right|^{2} & =\left|\left(\Phi_{n},(P(J) A)^{*} P(J) B \Phi_{n}\right)\right|^{2} \\
& \leq\left(\Phi_{n}, A^{*} P(J) A \Phi_{n}\right)\left(\Phi_{n}, B^{*} P(J) B \Phi_{n}\right) .
\end{aligned}
$$

We decompose the set $J$ as $J_{+} \cup J_{-}$, where $E>E_{n}$ for any $E \in J_{+}$and $E<E_{n}$ for any $E \in J_{-}$. Then we find that

$$
\begin{aligned}
\left(\Phi_{n}, A^{*} P(J) A \Phi_{n}\right)= & \left(\Phi_{n}, A^{*} P\left(J_{+}\right) A \Phi_{n}\right)+\left(\Phi_{n}, A^{*} P\left(J_{-}\right) A \Phi_{n}\right) \\
\leq & \left|\left(\Phi_{n}, A^{*} P\left(J_{+}\right) \frac{H_{\Lambda}-E_{n}}{D} P\left(J_{+}\right) A \Phi_{n}\right)\right| \\
& +\left|\left(\Phi_{n}, A^{*} P\left(J_{-}\right) \frac{H_{\Lambda}-E_{n}}{D} P\left(J_{-}\right) A \Phi_{n}\right)\right|
\end{aligned}
$$

On the other hand, we have

$$
\begin{aligned}
\left|\left(\Phi_{n}, A^{*} P\left(J_{ \pm}\right)\left(H_{\Lambda}-E_{n}\right) P\left(J_{ \pm}\right) A \Phi_{n}\right)\right| & =\left|\left(\Phi_{n}, A^{*} P\left(J_{ \pm}\right)\left[H_{\Lambda}, A\right] \Phi_{n}\right)\right| \\
& \leq\|A\|\left\|\left[H_{\Lambda}, A\right]\right\| \\
& \leq 2 \operatorname{rmh}(o N)^{2 m}
\end{aligned}
$$

where, to get the final bound, we used the identity

$$
\left[H_{\Lambda}, A\right]=\sum_{i=1}^{m} O_{\Lambda} P_{1} \ldots O_{\Lambda} P_{i-1}\left[H_{\Lambda}, O_{\Lambda}\right] P_{i} O_{\Lambda} P_{\imath+1} \ldots O_{\Lambda}
$$


and the bound

$$
\left\|\left[H_{\Lambda}, O_{\Lambda}\right]\right\| \leq 2 \operatorname{rhoN},
$$

which follows from the assumptions ii) and iii) of Sect. 2. By combining the bounds (3.21) and (3.22), we immediately see that

$$
\left(\Phi_{n}, A^{*} P(J) A \Phi_{n}\right) \leq \frac{4 r m h(o N)^{2 m}}{D}
$$

Similarly, one can prove the same inequality (with $m$ replaced with $2 k-m$ ) for $\left(\Phi_{n}, B^{*} P(J) B \Phi_{n}\right)$. Then the desired inequality (3.17) follows from the inequality (3.20).

Proof of Proposition 3.2. The properties (3.12)-(3.15) are trivial consequences of the construction. We shall prove (3.16). Let us write

$$
\begin{aligned}
\tilde{I} & :=\left[\widetilde{E}_{1}, \widetilde{E}_{2}-h N^{1 / 4}\right) \subset I_{1}=\left[\widetilde{E}_{1}, \widetilde{E}_{2}\right), \\
\tilde{I}_{\ell} & :=\left[\widetilde{E}_{\ell}+h N^{1 / 4}, \widetilde{E}_{\ell+1}-h N^{1 / 4}\right) \subset I_{\ell}=\left[\widetilde{E}_{\ell}, \widetilde{E}_{\ell+1}\right) \quad(\ell=2,3, \ldots, M-1), \\
\tilde{I}_{M} & :=\left[\widetilde{E}_{M}+h N^{1 / 4}, \widetilde{E}_{M+1}\right) \subset I_{M}=\left[\widetilde{E}_{M}, \widetilde{E}_{M+1}\right) .
\end{aligned}
$$

Since the projection operators $P\left(I_{\ell}\right)$ and $P\left(\tilde{I}_{\ell}\right)$ commute with the Boltzmann factor $e^{-\beta H_{\Lambda}}$, we have

$$
\begin{aligned}
& \left\langle\left(O_{\Lambda}\right)^{2 k}\right\rangle_{\Lambda}-\left\langle\left(\tilde{O}_{\Lambda}\right)^{2 k}\right\rangle_{\Lambda}=\sum_{\ell=1}^{M}\left\langle P\left(I_{\ell}\right)\left\{\left(O_{\Lambda}\right)^{2 k}-\left(\tilde{O}_{\Lambda}\right)^{2 k}\right\} P\left(I_{\ell}\right)\right\rangle_{\Lambda} \\
& =\sum_{\ell=1}^{M}\left\langle P\left(\tilde{I}_{\ell}\right)\left\{\left(O_{\Lambda}\right)^{2 k}-\left(\widetilde{O}_{\Lambda}\right)^{2 k}\right\} P\left(\tilde{I}_{\ell}\right)\right\rangle_{\Lambda} \\
& \quad+\sum_{\ell=1}^{M}\left\langle P\left(I_{\ell} \backslash \tilde{I}_{\ell}\right)\left\{\left(O_{\Lambda}\right)^{2 k}-\left(\tilde{O}_{\Lambda}\right)^{2 k}\right\} P\left(I_{\ell} \backslash \tilde{I}_{\ell}\right)\right\rangle_{\Lambda} .
\end{aligned}
$$

The second term in the right-hand side of (3.27) can be bounded as

$$
\begin{aligned}
& \left|\sum_{\ell=1}^{M}\left\langle P\left(I_{\ell} \backslash \tilde{I}_{\ell}\right)\left\{\left(O_{\Lambda}\right)^{2 k}-\left(\widetilde{O}_{\Lambda}\right)^{2 k}\right\} P\left(I_{\ell} \backslash \tilde{I}_{\ell}\right)\right\rangle_{\Lambda}\right| \\
& \quad \leq\left\|\left(O_{\Lambda}\right)^{2 k}-\left(\widetilde{O}_{\Lambda}\right)^{2 k}\right\| \sum_{\ell=1}^{M}\left\langle P\left(I_{\ell} \backslash \tilde{I}_{\ell}\right)\right\rangle_{\Lambda} \\
& \quad=\left\|\left(O_{\Lambda}\right)^{2 k}-\left(\widetilde{O}_{\Lambda}\right)^{2 k}\right\| \sum_{\ell=2}^{M}\left\langle P\left(\Delta J_{\ell}\right)\right\rangle_{\Lambda} \\
& \quad \leq 2(o N)^{2 k} \times 4 N^{-1 / 4},
\end{aligned}
$$

where we have used the assumption ii) and the bound (3.6). 
Now we rewrite the first term in the right hand side of (3.27) as

$$
\begin{aligned}
\sum_{\ell=1}^{M}\left\langle P\left(\tilde{I}_{\ell}\right)\left\{\left(O_{\Lambda}\right)^{2 k}-\left(\tilde{O}_{\Lambda}\right)^{2 k}\right\} P\left(\tilde{I}_{\ell}\right)\right\rangle_{\Lambda} \\
=\sum_{\ell=1}^{M}\left\{\left\langle P\left(\tilde{I}_{\ell}\right) O_{\Lambda}\left(P\left(I_{\ell}\right)+P\left(\bar{I}_{\ell}\right)\right) O_{\Lambda}\left(P\left(I_{\ell}\right)+P\left(\bar{I}_{\ell}\right)\right) \ldots O_{\Lambda} P\left(\tilde{I}_{\ell}\right)\right\rangle_{\Lambda}\right. \\
\quad-\left\langle P\left(\tilde{I}_{\ell}\right) O_{\Lambda} P\left(I_{\ell}\right) O_{\Lambda} P\left(I_{\ell}\right) \ldots O_{\Lambda}\left(P\left(\tilde{I}_{\ell}\right)\right\rangle_{\Lambda}\right\} \\
=\sum_{\substack{\ell=1 \\
\hat{I}_{\ell}^{(j)}=I_{\ell} \text { or } \bar{I}_{\ell} \\
(\jmath=1, \ldots, 2 k-1)}}^{\prime}\left\langle P\left(\tilde{I}_{\ell}\right) O_{\Lambda} P\left(\hat{I}_{\ell}^{(1)}\right) O_{\Lambda} P\left(\hat{I}_{\ell}^{(2)}\right) \ldots P\left(\hat{I}_{\ell}^{(2 k-1)}\right) O_{\Lambda} P\left(\tilde{I}_{\ell}\right)\right\rangle_{\Lambda} .
\end{aligned}
$$

where $\bar{I}:=I \backslash I_{\ell}$, and the second sum $\sum^{\prime}$ in the right-hand side is taken over all the assignments $\hat{I}_{\ell}^{(\jmath)}=I_{\ell}$ or $\bar{I}_{\ell}$ for $j=1,2, \ldots, 2 k-1$, except for one assignment $\hat{I}_{\ell}^{(j)}=I_{\ell}$ for all $j$. Thus the sum ranges over $2^{2 k-1}-1$ different assignments.

Note that, in each term in the right-hand side of (3.29), there is at least one $j(=1, \ldots, 2 k-1)$ such that $\hat{I}_{\ell}^{(j)}$ is $\bar{I}_{\ell}$. Thus we can make use of Lemma 3.3 by letting the index $n$ be such that $E_{n} \in \tilde{I}_{\ell}, J=\bar{I}_{\ell}$, and $D=h N^{1 / 4}$, to get

$$
\begin{aligned}
& \left\langle P\left(\tilde{I}_{\ell}\right) O_{\Lambda} P\left(\hat{I}_{\ell}^{(1)}\right) O_{\Lambda} P\left(\hat{I}_{\ell}^{(2)}\right) \ldots P\left(\hat{I}_{\ell}^{(2 k-1)}\right) O_{\Lambda} P\left(\tilde{I}_{\ell}\right)\right\rangle_{\Lambda} \\
& =\sum_{n: E_{n} \in \tilde{I}_{\ell}}\left(\Phi_{n}, O_{\Lambda} P\left(\hat{I}_{\ell}^{(1)}\right) O_{\Lambda} P\left(\hat{I}_{\ell}^{(2)}\right) \ldots P\left(\hat{I}_{\ell}^{(2 k-1)}\right) O_{\Lambda} \Phi_{n}\right) \times \frac{e^{-\beta E_{n}}}{Z_{\Lambda}} \\
& \leq \sum_{n: E_{n} \in \tilde{I}_{\ell}} \frac{4 r k h(o N)^{2 k}}{h N^{1 / 4}} \times \frac{e^{-\beta E_{n}}}{Z_{\Lambda}} \\
& =4 r k(o N)^{2 k} \times N^{-1 / 4} \times\left\langle P\left(\tilde{I}_{\ell}\right)\right\rangle_{\Lambda} .
\end{aligned}
$$

Substituting (3.30) into (3.29), we get

$$
\begin{aligned}
& \left|\sum_{\ell=1}^{M}\left\langle P\left(\tilde{I}_{\ell}\right)\left\{\left(O_{\Lambda}\right)^{2 k}-\left(\tilde{O}_{\Lambda}\right)^{2 k}\right\} P\left(\tilde{I}_{\ell}\right)\right\rangle_{\Lambda}\right| \\
& \quad \leq \sum_{\ell=1}^{M}\left(2^{2 k-1}-1\right) \times 4 r k(o N)^{2 k} \times N^{-1 / 4} \times\left\langle P\left(\tilde{I}_{\ell}\right)\right\rangle_{\Lambda} \\
& \quad \leq\left(2^{2 k-1}-1\right) \times 4 r k(o N)^{2 k} \times N^{-1 / 4}
\end{aligned}
$$

By substituting the bounds (3.28) and (3.31) into the decomposition (3.27), and using $r k \geq 2$, we get the desired inequality (3.16).

It is crucial that we have defined the decomposition of the interval $I=I_{1} \cup \ldots \cup I_{M}$ not by simply dividing $I$ into subintervals of equal length, but by carefully choosing the boundaries $\left\{\widetilde{E}_{\ell}\right\}$ according to Lemma 3.1. Without this extra care, one might encounter a situation where the energy eigenvalues have bulk degeneracy exactly at 
a boundary value $\widetilde{E}_{\ell}$. Then the contribution of the boundary term treated in (3.28) is no longer small.

The present definitions of the commuting operators $\widetilde{H}_{\Lambda}$ and $\widetilde{O}_{\Lambda}$ depend explicitly on the value of $\beta$, or more precisely, on the probability distribution of energy. The discussion in the previous paragraph suggests, however, that one might be able to find a decomposition of $I$ which depends only on the spectrum of $H_{\Lambda}$, and not on the inverse temperature $\beta$. Unfortunately, we still do not know how to define such a decomposition.

\section{Probability Distribution of the Approximate Magnetization in Zero-Field}

In the present section, we derive a bound on the probability distribution of the approximate (staggered) magnetization, i.e., the eigenvalue of the approximate order operator $\widetilde{O}_{\Lambda}$ constructed in the previous section. The bound plays a crucial role in the proof of the main Theorem 2.1. The argument here is a modification of Griffith's [10]. Recall that the inverse temperatur $\beta$ and the lattice $\Lambda$ are still fixed.

Since the operators $\widetilde{H}_{\Lambda}$ and $\widetilde{O}_{\Lambda}$ commute with each other [see (3.12) in Proposition 3.2], one can take an orthonormal basis $\left\{\Psi_{n, l}\right\}_{n, l}$ in which both of them are diagonal. We write their eigenvalues as

$$
\widetilde{O}_{\Lambda} \Psi_{n, l}=\widetilde{M}_{n, l} \Psi_{n, l}
$$

and

$$
\widetilde{H}_{\Lambda} \Psi_{n, l}=\widetilde{E}_{n} \Psi_{n, l}
$$

Let us define

$$
\varrho_{n, l}:=\frac{\left(\Psi_{n, l}, e^{-\beta H_{\Lambda}} \Psi_{n, l}\right)}{\operatorname{Tr}\left[e^{-\beta H_{\Lambda}}\right]},
$$

which is the probability density for the approximate (staggered) magnetization $\widetilde{M}_{n, l}$, with respect to the Boltzmann weight determined by the "true" Hamiltonion $H_{\Lambda}$. We also define

$$
\operatorname{Prob}\left(\left|\widetilde{M}_{n, l}\right| \geq N m\right):=\sum_{(n, l):\left|\widetilde{M}_{n, l}\right| \geq N m} \varrho_{n, l},
$$

which is the probability to find $\widetilde{M}_{n, l}$ with $\left|\widetilde{M}_{n, l}\right| \geq N m$.

We now define

$$
m_{\mathrm{s}}^{*}:=-\lim _{B \downarrow o} \frac{f(B)-f(0)}{B},
$$

which exists since the free energy $f(B)$ is concave [14]. The concavity of $f(B)$ also implies

$$
-\frac{f_{\Lambda}(B)-f_{\Lambda}(0)}{B} \leq-\frac{\partial}{\partial B} f_{\Lambda}(B)=\frac{1}{N}\left\langle O_{\Lambda}\right\rangle_{\Lambda}(B)
$$

By letting first $\Lambda \uparrow \mathbf{Z}^{d}$ and then $B \downarrow 0$, the bound (4.6) reduces to

$$
m_{\mathrm{s}}^{*} \leq m_{\mathrm{s}} \text {. }
$$

Then the main result of the present section is the following. 
Proposition 4.1. For any $m>m_{\mathrm{s}}^{*}$, there exists a $\delta>0$ (depending on $m$ ) such that

$$
\operatorname{Prob}\left(\left|\widetilde{M}_{n, l}\right| \geq N m\right) \leq 2 \times e^{-\beta N \delta} \times e^{\beta h \sqrt{N}},
$$

for sufficiently large $N$ (i.e., the number of sites in $\Lambda$ ).

We shall prove Proposition 4.1 in the following. We first note that, for any projection operator $P_{\Lambda}$,

$$
1 \geq \frac{\operatorname{Tr}\left[P_{\Lambda} e^{-\beta N \widetilde{H}_{\Lambda}(B)}\right]}{\operatorname{Tr}\left[e^{-\beta N \widetilde{H}_{\Lambda}(B)}\right]}
$$

where

$$
\widetilde{H}_{\Lambda}(B):=\widetilde{H}_{\Lambda}-B O_{\Lambda}
$$

We take

$$
Q(V):=\sum_{(n, l) \in V} Q_{n, l}
$$

as $P_{\Lambda}$ in (4.9), where $Q_{n, l}$ is the projection operator into the eigenstate $\Psi_{n, l}$, and $V$ is a set of indices $(n, l)$. Then the inequality (4.9) becomes

$$
1 \geq e^{\beta N \tilde{f}_{\Lambda}(B)} \times \sum_{(n, l) \in V}\left(\Psi_{n, l}, e^{-\beta \widetilde{H}_{\Lambda}(B)} \Psi_{n, l}\right),
$$

where

$$
\tilde{f}_{\Lambda}(B):=-\frac{1}{\beta N} \log \operatorname{Tr} e^{-\beta \widetilde{H}_{\Lambda}(B)}
$$

By applying the Jensen inequality

$$
\left(\Psi_{n, l}, e^{-\beta \widetilde{H}_{\Lambda}^{(B)}} \Psi_{n, l}\right) \geq \exp \left[-\beta\left(\Psi_{n, l}, \widetilde{H}_{\Lambda}(B) \Psi_{n, l}\right)\right],
$$

to the right-hand side of (4.12), we get

$$
\begin{aligned}
1 & \geq e^{\beta N \tilde{f}_{\Lambda}(B)} \sum_{(n, l) \in V} \exp \left[-\beta\left(\Psi_{n, l}, \widetilde{H}_{\Lambda}(B) \Psi_{n, l}\right)\right] \\
& =e^{\beta N \tilde{f}_{\Lambda}(B)} \sum_{(n, l) \in V} e^{-\beta \widetilde{E}_{n}} \times e^{\beta B \widetilde{M}_{n, l}}
\end{aligned}
$$

where we used (3.15) in Proposition 3.2, (4.1) and (4.2).

Note that we have

$$
e^{-\beta \widetilde{E}_{n}} \geq\left(\Psi_{n, l}, e^{-\beta H_{\Lambda}(B)} \Psi_{n, l}\right)
$$

from (3.13) in Proposition 3.2 and (4.2). We also need the following lemma to evaluate the right-hand side of (4.15),

\section{Lemma 4.2.}

$$
\tilde{f}_{\Lambda}(B) \geq f_{\Lambda}(B)-h N^{-\frac{1}{2}}
$$


Proof. Note that

$$
\begin{aligned}
\left|\tilde{f}_{\Lambda}(B)-f_{\Lambda}(B)\right| & =\frac{1}{\beta N}\left|\log \operatorname{Tr} e^{-\beta \widetilde{H}_{\Lambda}(B)}-\log \operatorname{Tr} e^{-\beta H_{\Lambda}(B)}\right| \\
& =\frac{1}{\beta N}\left|\int_{0}^{1} d \lambda \frac{d}{d \lambda} \log \operatorname{Tr} \exp \left[-\beta H_{\Lambda}(B)-\lambda \beta\left(\widetilde{H}_{\Lambda}-H_{\Lambda}\right)\right]\right| \\
& =\frac{1}{N} \mid \int_{0}^{1} d \lambda \frac{\operatorname{Tr}\left(\widetilde{H}_{\Lambda}-H_{\Lambda}\right) \exp \left[-\beta H_{\Lambda}(B)-\lambda \beta\left(\widetilde{H}_{\Lambda}-H_{\Lambda}\right)\right]}{\operatorname{Tr} \exp \left[-\beta H_{\Lambda}(B)-\lambda \beta\left(\widetilde{H}_{\Lambda}-H_{\Lambda}\right)\right]} \\
& \leq \frac{1}{N}\left\|\widetilde{H}_{\Lambda}-H_{\Lambda}\right\| .
\end{aligned}
$$

Therefore, the lemma follows from (3.14) in Proposition 3.2.

By (4.15), (4.16) and the above lemma, we obtain the inequality

$$
1 \geq \exp \left[\beta N\left\{f_{\Lambda}(B)-f_{\Lambda}(0)\right\}\right] \times e^{-\beta h \sqrt{N}} \times \sum_{(n, l) \in K} e^{\beta B \widetilde{M}_{n, l}} \times \varrho_{n, l},
$$

where $\varrho_{n, l}$ is defined in (4.3). Then from the definition (4.4) and the inequality (4.19), we have

$$
\begin{aligned}
\operatorname{Prob}\left(\left|\widetilde{M}_{n, l}\right| \geq N m\right) \leq 2 & \times e^{-\beta B N m} \\
& \times \exp \left[-\beta N\left\{f_{\Lambda}(B)-f_{\lambda}(0)\right\}+\beta h N^{\frac{1}{2}}\right],
\end{aligned}
$$

for $B \geq 0$ and $m \geq 0$, where we used the symmetry (2.5) in Sect. 2 .

To prove the desired bound (4.8), we recall the following two facts.

First, for any $m>m_{\mathrm{s}}^{*}$, there exist $m^{\prime} \in\left[m_{\mathrm{s}}^{*}, m\right)$ and $B>0$ such that

$$
f(B)-f(0) \geq-m^{\prime} B .
$$

This fact was proved by Griffiths [10] from the concavity of the free energy $f(B)$. Secondly, from the assumption i) in Sect. 2 on the existence of a thermodynamic limit of the free energy, we find that, for any given $\delta>0$, there exists an integer $N_{0}$ such that

$$
\begin{aligned}
\left|f(B)-f_{\Lambda}(B)\right| & \leq \frac{\delta}{2} \\
\left|f(0)-f_{\Lambda}(0)\right| & \leq \frac{\delta}{2},
\end{aligned}
$$

for any $\Lambda$ with $|\Lambda| \geq N_{0}$ and for fixed $B \in \mathbf{R}$.

For a given $m>m_{\mathrm{s}}^{*}$, we take $\delta=\frac{1}{2} B\left(m-m^{\prime}\right)$, where $B$ and $m^{\prime}$ are chosen so that the bound (4.21) holds. By combining the bounds (4.21), (4.22) and (4.23), we get

$$
f_{\Lambda}(B)-f_{\Lambda}(0) \geq-m^{\prime} B-\delta
$$

for sufficiently large $N$.

The desired inequality (4.8) follows from the inequalities (4.20) and (4.24). 


\section{Proof of the Main Theorem 2.1}

Now we prove Theorem 2.1 stated in Sect. 2.

Take an arbitrary $m>m_{\mathrm{s}}^{*}$, where $m_{\mathrm{s}}^{*}$ defined in (4.5). Then, from (4.1) and (4.3), we have

$$
\begin{aligned}
\left\langle\left(\widetilde{O}_{\Lambda}\right)^{2 k}\right\rangle_{\Lambda} & =\frac{\operatorname{Tr}\left[\left(\widetilde{O}_{\Lambda}\right)^{2 k} e^{-\beta H_{\Lambda}}\right]}{\operatorname{Tr} e^{-\beta H_{\Lambda}}} \\
& =\sum_{(n, l)}\left(\widetilde{M}_{n, l}\right)^{2 k} \varrho_{n, l} \\
& =\sum_{(n, l):\left|\widetilde{M}_{n, l}\right| \geq N m}\left(\widetilde{M}_{n, l}\right)^{2 k} \varrho_{n, l}+\sum_{(n, l):\left|\widetilde{M}_{n, l}\right|<N m}\left(\widetilde{M}_{n, l}\right)^{2 k} \varrho_{n, l},
\end{aligned}
$$

for any positive integer $k$.

By the definition (4.4) and Proposition 4.1, we get

$$
\begin{aligned}
\frac{1}{N^{2 k}}\left\langle\left(\widetilde{O}_{\Lambda}\right)^{2 k}\right\rangle_{\Lambda} & \leq o^{2 k} \operatorname{Prob}\left(\left|\widetilde{M}_{n, l}\right| \geq N m\right)+m^{2 k} \\
& \leq 2 \times o^{2 k} e^{-\beta N \delta} \times e^{\beta h \sqrt{N}}+m^{2 k} .
\end{aligned}
$$

Combining the bound (5.2) with (3.16) in Proposition 3.2, we obtain

$$
\frac{1}{N^{2 k}}\left\langle\left(O_{\Lambda}\right)^{2 k}\right\rangle_{\Lambda}(0) \leq m^{2 k}+2 \times o^{2 k} e^{-\beta N \delta+\beta h \sqrt{N}}+4^{k+1} k r o^{2 k} N^{-1 / 4} .
$$

By taking the limits $N \uparrow \infty$ and $m \downarrow m_{\mathrm{s}}^{*}$, the inequality (5.3) reduces to the desired inequality (2.13) with the right-hand side replaced with $m_{\mathrm{s}}^{*}$. Since we have the bound (4.7), Theorem 2.1 has been proved.

\section{Consequence of the Symmetry}

In the present section, we prove a theorem which characterizes a state with an $S U(2)$ symmetry. The theorem is quite general, and can be applied to both the equilibrium states and the ground states. In the following $\langle\cdots\rangle_{\Lambda}$ stands for the expectation in either the equilibrium state with vanishing external magnetic field or the symmetric finite volume ground state $\Phi_{\Lambda}$. The similar result was proved by Dyson, Lieb and Simon [4] for the Heisenberg ferromagnets, where they made explicit use of the representation of angular momenta. Our extension here is based on a much more "classical" consideration.

Theorem 6.1. Let $\langle\cdots\rangle_{\Lambda}$ be a state (i.e., a normalized linear functional) on the operators of the quantum system on $\Lambda$. We assume that the state is invariant under $S U(2)$ transformation, in the sense that for any unitary transformation $U_{\Lambda}$ generated by the $S U(2)$ generators $X_{\Lambda}^{(1)}, X_{\Lambda}^{(2)}$ and $X_{\Lambda}^{(3)}$, we have

$$
\left\langle U_{\Lambda} A U_{\Lambda}^{*}\right\rangle_{\Lambda}=\langle A\rangle_{\Lambda}
$$

for any operator A. We also assume ii), iv) and v) in Sect. 2. Then we have

$$
\lim _{k \uparrow \infty} \lim _{N \uparrow \infty} \frac{1}{N}\left\langle\left(O_{\Lambda}^{(1)}\right)^{2 k}\right\rangle_{\Lambda}^{\frac{1}{2 k}} \geq \sqrt{3} \lim _{N \uparrow \infty} \frac{1}{N}\left\langle\left(O_{\Lambda}^{(1)}\right)^{2}\right\rangle_{\Lambda}^{\frac{1}{2}} .
$$


We start from lemmas. Let $\ell, m$ and $n$ be nonnegative integers, and let $k=$ $\ell+m+n$. Define

$$
P_{\ell, m, n}:=\frac{(2 k) !}{(2 \ell) !(2 m) !(2 n) !} \frac{1}{4 \pi} \int d \boldsymbol{\Omega} x^{2 \ell} y^{2 m} z^{2 n},
$$

where the integration is over the unit sphere $\left\{\boldsymbol{\Omega}=(x, y, z) \mid x^{2}+y^{2}+z^{2}=1\right\}$.

Lemma 6.2. We have

$$
P_{\ell, m, n}=\frac{k !}{\ell ! m ! n !} G_{k}
$$

where $G_{k} \leq 1$ and

$$
\lim _{k \uparrow \infty}\left(G_{k}\right)^{\frac{1}{2 k}}=1
$$

Proof. Let

$$
F(a, b, c):=\frac{1}{4 \pi} \int d \boldsymbol{\Omega}(a x+b y+c z)^{2 k}
$$

Then by expanding $(a x+b y+c z)^{2 k}$, we observe that

$$
P_{\ell, m, n}=\frac{1}{\ell ! m ! n !} \frac{\partial^{\ell}}{\partial\left(a^{2}\right)^{\ell}} \frac{\partial^{m}}{\partial\left(b^{2}\right)^{m}} \frac{\partial^{n}}{\partial\left(c^{2}\right)^{n}} F(a, b, c) .
$$

On the other hand, from the rotation invariance, we get

$$
\begin{aligned}
F(a, b, c) & =\frac{1}{4 \pi} \int d \boldsymbol{\Omega}\{(a, b, c) \cdot \boldsymbol{\Omega}\}^{2 k} \\
& =\frac{1}{4 \pi} \int d \boldsymbol{\Omega}\left\{\left(\sqrt{a^{2}+b^{2}+c^{2}}, 0,0\right) \cdot \boldsymbol{\Omega}\right\}^{2 k} \\
& =\left(a^{2}+b^{2}+c^{2}\right)^{k} G_{k},
\end{aligned}
$$

with

$$
G_{k}:=\frac{1}{4 \pi} \int d \boldsymbol{\Omega} x^{2 k}
$$

The relation (6.5) is trivial since the maximum value of $|x|$ in the support of the integral is 1. By substituting the identity (6.8) into the representation (6.7), we immediately get the desired identity (6.4).

Lemma 6.3. Let $i_{j}$ with $j=1, \ldots, 2 k$ be such that there are $2 \ell$ different indices $j$ with $i_{j}=1,2 m$ different $j$ with $i_{\jmath}=2$, and $2 n$ different $j$ with $i_{\jmath}=3$, where $k=\ell+m+n$. Then we have

$$
\begin{aligned}
& \left|\left\langle O_{\Lambda}^{\left(i_{1}\right)} O_{\Lambda}^{\left(i_{2}\right)} \cdots O_{\Lambda}^{\left(i_{2 k}\right)}\right\rangle_{\Lambda}-\left\langle\left(O_{\Lambda}^{(1)}\right)^{\ell}\left(O_{\Lambda}^{(2)}\right)^{m}\left(O_{\Lambda}^{(3)}\right)^{2 n}\left(O_{\Lambda}^{(2)}\right)^{m}\left(O_{\Lambda}^{(1)}\right)^{\ell}\right\rangle_{\Lambda}\right| \\
& \quad \leq 8 \operatorname{lmn} 2(o N)^{2 k} N^{-1} \\
& \quad \leq 2 k^{3}(o N)^{2 k} N^{-1} .
\end{aligned}
$$

Proof. We can get the second term from the first term in the left-hand side of (6.10) by exchanging neighboring operators at most $8 \ell m n$ times. By combining this observation with the bound $\left\|\left[O_{\Lambda}^{(\imath)}, O_{\Lambda}^{(\jmath)}\right]\right\| \leq 2 o^{2} N$, one immediately gets the desired inequality. 
Proof of Theorem 6.1. We first note that

$$
\begin{aligned}
& \left\langle\left\{\left(O_{\Lambda}^{(1)}\right)^{2}+\left(O_{\Lambda}^{(2)}\right)^{2}+\left(O_{\Lambda}^{(3)}\right)^{2}\right\}^{k}\right\rangle_{\Lambda} \\
& \leq \sum_{\ell, m, n \geq 0 ; \ell+m+n=k} \frac{k !}{\ell ! m ! n !}\left\langle\left(O_{\Lambda}^{(1)}\right)^{\ell}\left(O_{\Lambda}^{(2)}\right)^{m}\left(O_{\Lambda}^{(3)}\right)^{2 n}\left(O_{\Lambda}^{(2)}\right)^{m}\left(O_{\Lambda}^{(1)}\right)^{\ell}\right\rangle_{\Lambda} \\
& \quad+2 k^{3}(o N)^{2 k} N^{-1}
\end{aligned}
$$

where we have expanded the left-hand side, and used the bound (6.10) in each term of the expansion.

Let $(x, y, z)$ be a point on the unit sphere. From the $S U(2)$ invariance, one can find a (nonunique) unitary operator $U_{\Lambda}(x, y, z)$ such that

$$
U_{\Lambda}(x, y, z) O_{\Lambda}^{(1)} U_{\Lambda}^{*}(x, y, z)=x O_{\Lambda}^{(1)}+y O_{\Lambda}^{(2)}+z O_{\Lambda}^{(3)} .
$$

By using the $S U(2)$ invariance of the expectation, we get

$$
\begin{aligned}
& \left\langle\left(O_{\Lambda}^{(1)}\right)^{2 k}\right\rangle_{\Lambda}=\frac{1}{4 \pi} \int d \mathbf{\Omega}\left\langle\left(x O_{\Lambda}^{(1)}+y O_{\Lambda}^{(2)}+z O_{\Lambda}^{(3)}\right)^{2 k}\right\rangle_{\Lambda} \\
& \geq \sum_{\ell, m, n} P_{\ell, m, n}\left\{\left\langle\left(O_{\Lambda}^{(1)}\right)^{\ell}\left(O_{\Lambda}^{(2)}\right)^{m}\left(O_{\Lambda}^{(3)}\right)^{2 n}\left(O_{\Lambda}^{(2)}\right)^{m}\left(O_{\Lambda}^{(1)}\right)^{\ell}\right\rangle_{\Lambda}-2 k^{3}(o N)^{2 k} N^{-1}\right\} \\
& =G_{k} \sum_{\ell, m, n} \frac{k !}{\ell ! m ! n !}\left\{\left\langle\left(O_{\Lambda}^{(1)}\right)^{\ell}\left(O_{\Lambda}^{(2)}\right)^{m}\left(O_{\Lambda}^{(3)}\right)^{2 n}\left(O_{\Lambda}^{(2)}\right)^{m}\left(O_{\Lambda}^{(1)}\right)^{\ell}\right\rangle_{\Lambda}-2 k^{3}(o N)^{2 k} N^{-1}\right\} \\
& \geq G_{k}\left\langle\left\{\left(O_{\Lambda}^{(1)}\right)^{2}+\left(O_{\Lambda}^{(2)}\right)^{2}+\left(O_{\Lambda}^{(3)}\right)^{2}\right\}^{k}\right\rangle_{\Lambda}-4 k^{3}(o N)^{2 k} N^{-1} \\
& \geq G_{k}\left\langle\left(O_{\Lambda}^{(1)}\right)^{2}+\left(O_{\Lambda}^{(2)}\right)^{2}+\left(O_{\Lambda}^{(3)}\right)^{2}\right\rangle_{\Lambda}^{k}-4 k^{3}(o N)^{2 k} N^{-1}
\end{aligned}
$$

where the sums are taken over integers $\ell, m, n$ with $\ell+m+n=k$. We have used (6.10), (6.7) and (6.11). The final bound follows from the Hölder inequality.

Thus we find that

$$
\frac{1}{N}\left\langle\left(O_{\Lambda}^{(1)}\right)^{2 k}\right\rangle_{\Lambda}^{\frac{1}{2 k}} \geq \frac{1}{N}\left\{3^{k} G_{k}\left\langle\left(O_{\Lambda}^{(1)}\right)^{2}\right\rangle_{\Lambda}^{k}-4 k^{3}(o N)^{2 k} N^{-1}\right\}^{\frac{1}{2 k}}
$$

Note that we can assume

$$
\lim _{N \uparrow \infty} \frac{1}{N}\left\langle\left(O_{\Lambda}^{(1)}\right)^{2}\right\rangle_{\Lambda}^{\frac{1}{2}}>0
$$

since the desired inequality (6.2) is trivial when the limit is vanishing. Then, by letting $N \uparrow \infty$ and then $k \uparrow \infty$, the inequality (6.14) reduces to the desired (6.2).

Remark. Theorem 6.1 can easily extended to systems with symmetry other than $S U(2)$. When a system has an $S O(n)$ invariance, one gets a bound similar to (6.2) with the factor $\sqrt{3}$ replaced with $\sqrt{n}$. (In the above, we have actually made use of the $S O(3)$ invariance, rather than the full $S U(2)$ invariance.) The most important of these extensions are the models with an $S O(2) \cong U(1)$ invariance, where we get a factor $\sqrt{2}$. Such models can be found in quantum antiferromagnets with an XY-like anisotropy, or the electron pair condensation problems in lattice electron systems. 


\section{Symmetry Breaking in Ground States}

In the present section, which is somewhat independent from the rest of the paper, we shall focus on the problem of symmetry breaking in ground states. Since our previous theorems are valid for any finite temperatures, they also have implications on the ground states obtaining by letting $\beta \rightarrow \infty$ after taking the infinite volume limit. We stress that these are the states which have direct relevance to properties of very large physical systems at low temperatures.

There has been, however, considerable effort to study ground states of finite systems (i.e., the $\beta \rightarrow \infty$ limit taken before the infinite volume limit) and the properties of their infinite volume limits. (See $[5-9,11,15]$ and the references therein.) We shall prove results similar to the previous ones for such ground states. One of the most interesting points in the theory of the present section is the explicit construction of the state (7.23) which exhibits explicit symmetry breaking with rather large order parameter.

The main result of the present section is an improvement of the theorem of Kaplan, Horsch and von der Linden [11]. The proof of our main theorem is also based on their variational argument.

Let $\Phi_{\Lambda}$ and $\Phi_{\Lambda}(B)$ be ground stated of the Hamiltonians (2.2) and (2.6), respectively. We define the long range order parameter in the ground state $\Phi_{\Lambda}$ as

$$
\sigma:=\lim _{\Lambda \uparrow \mathbf{Z}^{d}} \sqrt{\frac{1}{N^{2}}\left(\Phi_{\Lambda},\left(O_{\Lambda}\right)^{2} \Phi_{\Lambda}\right)},
$$

where we take a subsequence if necessary. We assume

i') $U_{\Lambda} \Phi_{\Lambda}=\Phi_{\Lambda}$, for $U_{\Lambda}$ used in Sect. 2. (See (2.3) and (2.5).)

Kaplan, Horsch and von der Linden [11] proved the following.

Theorem 7.1 (Kaplan, Horsch and von der Linden) [11]. Consider an arbitrary sequence of models satisfying the abover $\left.\mathrm{i}^{\prime}\right)$ and the assumptions ii), iii) in Sect. 2. Then we have

$$
\liminf _{B \downarrow 0} \liminf _{\Lambda \uparrow \mathbf{Z}^{d}} \frac{1}{N}\left(\Phi_{\Lambda}(B), O_{\Lambda} \Phi_{\Lambda}(B)\right) \geq \sigma
$$

An extra assumption needed in the improvement is

$\left.\mathrm{i}^{\prime \prime}\right)$ The ground state $\Phi_{\Lambda}$ is $S U(2)$ invariant, i.e., we have $X_{\Lambda}^{(i)} \Phi_{\Lambda}=0$ for $i=1,2,3$. Then we have the following.

Corollary 7.2. Assume that the conditions for Theorem 7.1 are valid, and we further have the $S U(2)$ invariance as in the above $\left.\mathrm{i}^{\prime \prime}\right)$ and the assumptions iv) and v) in Sect. 2. Then we have

$$
\liminf _{B \downarrow 0} \liminf _{\Lambda \uparrow \mathbf{Z}^{d}} \frac{1}{N}\left(\Phi_{\Lambda}(B), O_{\Lambda}^{(1)} \Phi_{\Lambda}(B)\right) \geq \sqrt{3} \sigma .
$$

Note that the discussions in Remark 3 of Sect. 1 applies to the infinite volume ground states as well. We thus believe that the bound (7.3) is saturated in "natural" systems with an $S U(2)$ invariance.

Let us first reproduce the proof of Theorem 7.1. 
Proof of Theorem 7.1 [11]. Let $\Psi_{\Lambda}$ be an arbitrary normalized state on the lattice $\Lambda$. Since $\Phi_{\Lambda}(B)$ is the normalized ground state of the Hamiltonian $H_{\Lambda}(B)$, we have

$$
\left(\Phi_{\Lambda}(B), H_{\Lambda}(B) \Phi_{\Lambda}(B)\right) \leq\left(\Psi_{\Lambda}, H_{\Lambda}(B), \Psi_{\Lambda}\right) .
$$

By substituting the definition (2.6) of $H_{\Lambda}(B)$, the inequality (7.4) reduces to the lower bound for the (staggered) magnetization as

$$
\begin{aligned}
\frac{1}{N}\left(\Phi_{\Lambda}(B), O_{\Lambda} \Phi_{\Lambda}(B)\right) \geq & \frac{1}{N}\left(\Psi_{\Lambda}, O_{\Lambda} \Psi_{\Lambda}\right) \\
& +\frac{1}{N B}\left\{\left(\Phi_{\Lambda}(B), H_{\Lambda} \Phi_{\Lambda}(B)\right)-\left(\Psi_{\Lambda}, H_{\Lambda} \Psi_{\Lambda}\right)\right\} \\
\geq & \frac{1}{N}\left(\Psi_{\Lambda}, O_{\Lambda} \Psi_{\Lambda}\right)+\frac{1}{N B}\left\{E_{0}-\left(\Psi_{\Lambda}, H_{\Lambda} \Psi_{\Lambda}\right)\right\}
\end{aligned}
$$

where $E_{0}$ denotes the ground state energy of the Hamiltonian $H_{\Lambda}$ under vanishing external field.

We choose the variational state $\Psi_{\Lambda}$ as

$$
\Psi_{\Lambda}=\frac{1}{\sqrt{2}}\left(\Phi_{\Lambda}+\frac{O_{\Lambda} \Phi_{\Lambda}}{\left\|O_{\Lambda} \Phi_{\Lambda}\right\|}\right) .
$$

We note that the symmetry requirement (2.5) and the assumption $i^{\prime}$ ) imply that $\left(\Phi_{\Lambda},\left(O_{\Lambda}\right)^{\ell} \Phi_{\Lambda}\right)=0$ for any positive odd integer $\ell$. We find that

$$
\left(\Psi_{\Lambda}, O_{\Lambda} \Psi_{\Lambda}\right)=\frac{\left(\Phi_{\Lambda},\left(O_{\Lambda}\right)^{2} \Phi_{\Lambda}\right)}{\left\|O_{\Lambda} \Phi_{\Lambda}\right\|}=\sigma_{\Lambda} N
$$

where we wrote

$$
\sigma_{\Lambda}:=\frac{1}{N} \sqrt{\left(\Phi_{\Lambda},\left(O_{\Lambda}\right)^{2} \Phi_{\Lambda}\right)}
$$

The variational energy of the state $\Psi_{\Lambda}$ can be evaluated as follows.

$$
\begin{aligned}
\left(\Psi_{\Lambda}, H_{\Lambda} \Psi_{\Lambda}\right)-E_{0} & =\frac{1}{2}\left(\Phi_{\Lambda}, H_{\Lambda} \Phi_{\Lambda}\right)+\frac{\left(\Phi_{\Lambda}, O_{\Lambda} H_{\Lambda} O_{\Lambda} \Phi_{\Lambda}\right)}{2\left(\Phi_{\Lambda},\left(O_{\Lambda}\right)^{2} \Phi_{\Lambda}\right)}-E_{0} \\
& =\frac{2\left(\Phi_{\Lambda}, O_{\Lambda} H_{\Lambda} O_{\Lambda} \Phi_{\Lambda}\right)-\left(\Phi_{\Lambda},\left(O_{\Lambda}\right)^{2} H_{\Lambda} \Phi_{\Lambda}\right)-\left(\Phi_{\Lambda}, H_{\Lambda}\left(O_{\Lambda}\right)^{2} \Phi_{\Lambda}\right)}{4\left(\Phi_{\Lambda},\left(O_{\Lambda}\right)^{2} \Phi_{\Lambda}\right)} \\
& =\frac{\left(\Phi_{\Lambda},\left[O_{\Lambda}\left[H_{\Lambda}, O_{\Lambda}\right]\right] \Phi_{\Lambda}\right)}{4\left(\Phi_{\Lambda},\left(O_{\Lambda}\right)^{2} \Phi_{\Lambda}\right)} \\
& \leq \frac{r^{2} h o^{2}}{\left(\sigma_{\Lambda}\right)^{2}} N^{-1} .
\end{aligned}
$$

We have used the assumptions ii) and iii) in Sect. 2 to evaluate the double commutator. As far as we know, the above energy estimate was first noted explicitly by Horsch and von der Linden [15].

By substituting (7.9) and (7.7) into the variational estimate (7.5), we get

$$
\frac{1}{N}\left(\Phi_{\Lambda}(B), O_{\Lambda} \Phi_{\Lambda}(B)\right) \geq \sigma_{\Lambda}-\frac{r^{2} o^{2} h}{\left(\sigma_{\Lambda}\right)^{2} B N^{2}} .
$$

By first letting $N \uparrow \infty$ and then $B \downarrow 0$ in the above inequality, we get the desired inequality (7.2). 
Our Corollary 7.2 is based on the following Theorem 7.3. The theorem can be proved only assuming a $U(1)$ invariance. Since this allows one to apply the theorem to a larger class of models (including, for example, the electron pair condensation problems in lattice electron systems), we shall make the condition explicit.

Here we only need one generator $X_{\Lambda}^{(3)}$ and two component order operator $\left(O_{\Lambda}^{(1)}, O_{\Lambda}^{(2)}\right)$. We assume that the conditions iv), v) and vi) in Sect. 2 are satisfied for $O_{\Lambda}^{(1)}, O_{\Lambda}^{(2)}$ and $X_{\Lambda}^{(3)}$, and we have $X_{\Lambda}^{(3)} \Phi_{\Lambda}=0$.

Theorem 7.3. Assume that the conditions for Theorem 7.1 are valid, and we further have the U(1) invariance as discussed above. Also assume that $\sigma$ defined in (7.1) is nonvanishing. Then we have

$$
\begin{gathered}
\underset{B \downarrow 0}{\liminf } \liminf _{\Lambda \uparrow \mathbf{Z}^{d}} \frac{1}{N}\left(\Phi_{\Lambda}(B), O_{\Lambda}^{(1)} \Phi_{\Lambda}(B)\right) \\
\geq \lim _{k \uparrow \infty} \lim _{N \uparrow \infty} \frac{1}{N}\left(\Phi_{\Lambda},\left(O_{\Lambda}^{(1)}\right)^{2 k} \Phi_{\Lambda}\right)^{\frac{1}{2 k}} .
\end{gathered}
$$

Proof of Corollary 7.2, given Theorem 7.3. Since the desired inequality (7.3) is trivial when $\sigma=0$, we assume $\sigma \neq 0$. Then, by combining Theorem 7.3 with Theorem 6.1, one immediately gets Corollary 7.2.

Before proving Theorem 7.3, we state some technical estimates needed in the proof. Again we write

$$
\sigma_{\Lambda}:=\frac{1}{N} \sqrt{\left(\Phi_{\Lambda},\left(O_{\Lambda}^{(1)}\right)^{2} \Phi_{\Lambda}\right)}
$$

We also introduce

$$
O_{\Lambda}^{ \pm}=O_{\Lambda}^{(1)} \pm i O_{\Lambda}^{(2)}
$$

These operators satisfy the commutation relations

$$
\left[O_{\Lambda}^{ \pm}, X_{\Lambda}^{(3)}\right]= \pm O_{\Lambda}^{ \pm}
$$

Lemma 7.4. Under the assumptions of Theorem 7.3, we have

$$
\left(\sigma_{\Lambda} N\right)^{2 n} \leq\left(\Phi_{\Lambda},\left(O_{\Lambda}^{(1)}\right)^{2 n} \Phi_{\Lambda}\right) \leq(o N)^{2 n},
$$

for any positive integer $n$.

Proof. We use the Schwarz inequality to get

$$
\begin{aligned}
\left(\Phi_{\Lambda},\left(O_{\Lambda}^{(1)}\right)^{2 n-2} \Phi_{\Lambda}\right)^{2} & =\left(\Phi_{\Lambda},\left(O_{\Lambda}^{(1)}\right)^{n}\left(O_{\Lambda}^{(1)}\right)^{n-2} \Phi_{\Lambda}\right)^{2} \\
& \leq\left(\Phi_{\Lambda},\left(O_{\Lambda}^{(1)}\right)^{2 n} \Phi_{\Lambda}\right)\left(\Phi_{\Lambda},\left(O_{\Lambda}^{(1)}\right)^{2 n-4} \Phi_{\Lambda}\right)
\end{aligned}
$$

By rearranging the inequality, we see that

$$
\frac{\left(\Phi_{\Lambda},\left(O_{\Lambda}^{(1)}\right)^{2 n} \Phi_{\Lambda}\right)}{\left(\Phi_{\Lambda},\left(O_{\Lambda}^{(1)}\right)^{2 n-2} \Phi_{\Lambda}\right)} \geq \frac{\left(\Phi_{\Lambda},\left(O_{\Lambda}^{(1)}\right)^{2 n-2} \Phi_{\Lambda}\right)}{\left(\Phi_{\Lambda},\left(O_{\Lambda}^{(1)}\right)^{2 n-4} \Phi_{\Lambda}\right)} \geq \cdots \geq\left(\sigma_{\Lambda} N\right)^{2} .
$$

The lower bound in (7.15) follows by multiplying together the inequality (7.17). The upper bound in (7.15) is a trivial consequence of the assumption ii) of Sect. 2.

Lemma 7.5. Under the assumptions of Theorem 7.3, we have

$$
\left|\left(\Phi_{\Lambda},\left(O_{\Lambda}^{-}\right)^{n}\left(O_{\Lambda}^{+}\right)^{n} \Phi_{\Lambda}\right)-b_{n}\left(\Phi_{\Lambda},\left(O_{\Lambda}^{(1)}\right)^{2 n} \Phi_{\Lambda}\right)\right| \leq 8 n^{2}(o N)^{2 n} N^{-1}
$$


for any positive integer $n$. The coefficient $b_{n}$ is

$$
b_{n}:=4^{n} \frac{(n !)^{2}}{(2 n) !}=\frac{(2 n) ! !}{(2 n-1) ! !} \geq 1 .
$$

The same inequality as (7.18) with $O_{\Lambda}^{-}$and $O_{\Lambda}^{+}$interchanged is also valid.

Proof. To prove (7.18), we note that (7.13) and (7.14) imply

$$
\begin{aligned}
\left(\Phi_{\Lambda},\left(O_{\Lambda}^{(1)}\right)^{2 n} \Phi_{\Lambda}\right) & =\left(\Phi_{\Lambda},\left(\frac{O_{\Lambda}^{(+)}+O_{\Lambda}^{-}}{2}\right)^{2 n} \Phi_{\Lambda}\right) \\
& =\frac{1}{4^{n}} \sum\left(\Phi_{\Lambda}, O_{\Lambda}^{ \pm} O_{\Lambda}^{ \pm} \ldots O_{\Lambda}^{ \pm} \Phi_{\Lambda}\right),
\end{aligned}
$$

where the sum is taken over all the alignments of $n$ copies of $O_{\Lambda}^{-}$and $n$ copies of $O_{\Lambda}^{+}$. There are $(2 n) ! /(n !)^{2}$ distinct alignments. Then the desired estimate (7.18) follows from the bound

$$
\max \left|\left(\Phi_{\Lambda}, O_{\Lambda}^{ \pm} O_{\Lambda}^{ \pm} \ldots O_{\Lambda}^{ \pm} \Phi_{\Lambda}\right)-\left(\Phi_{\Lambda},\left(O_{\Lambda}^{-}\right)^{n}\left(O_{\Lambda}^{+}\right)^{n} \Phi_{\Lambda}\right)\right| \leq 8 n^{2}(o N)^{2 n} N^{-1},
$$

where the maximum is taken over all the allowed alignments of operators. To show (7.21) one only has to note that one can rearrange alignments of the $2 n$ operators by exchanging neighboring operators at most $2 n$ times. Since we have

$$
\left\|\left[O_{\Lambda}^{-}, O_{\Lambda}^{+}\right]\right\|=\left\|2\left[O_{\lambda}^{(1)}, O_{\Lambda}^{(2)}\right]\right\| \leq 4 o^{2} N
$$

from the assumptions ii) and iv), we get (7.21).

Proof of Theorem 7.3. We choose our variational state as

$$
\Psi_{\Lambda}=\frac{1}{\sqrt{2 k+1}}\left\{\Phi_{\Lambda}+\sum_{n=1}^{k}\left(\frac{\left(O_{\Lambda}^{+}\right)^{n} \Phi_{\Lambda}}{\left\|\left(O_{\Lambda}^{+}\right)^{n} \Phi_{\Lambda}\right\|}+\frac{\left(O_{\Lambda}^{-}\right)^{n} \Phi_{\Lambda}}{\left\|\left(O_{\Lambda}^{-}\right)^{n} \Phi_{\Lambda}\right\|}\right)\right\} .
$$

By combining the lower bound in (7.15) with the bound (7.18), one can easely verify that the state (7.23) is well defined for sufficiently large $N$, with $k$ fixed. Since the states in the sum of (7.23) are orthogonal to each other, the state $\Psi_{\Lambda}$ is normalized. As before the variational energy of status $\Psi_{\Lambda}$ can be evaluated as

$$
\begin{aligned}
& \left(\Psi_{\Lambda}, H_{\Lambda} \Psi_{\Lambda}\right)-E_{0} \\
& =\frac{1}{2 k+1} \sum_{n=1}^{k}\left\{\frac{\left(\Phi_{\Lambda},\left(O_{\Lambda}^{-}\right)^{n} H_{\Lambda}\left(O_{\Lambda}^{+}\right)^{n} \Phi_{\Lambda}\right)}{\left\|\left(O_{\Lambda}^{+}\right)^{n} \Phi_{\Lambda}\right\|^{2}}+\frac{\left(\Phi_{\Lambda},\left(O_{\Lambda}^{+}\right)^{n} H_{\Lambda}\left(O_{\Lambda}^{-}\right)^{n} \Phi_{\Lambda}\right)}{\left\|\left(O_{\Lambda}^{-}\right)^{n} \Phi_{\Lambda}\right\|^{2}}-2 E_{0}\right\} \\
& =\frac{1}{2 k+1} \sum_{n=1}^{k}\left\{\frac{\left(\Phi_{\Lambda},\left(O_{\Lambda}^{-}\right)^{n}\left[H_{\Lambda},\left(O_{\Lambda}^{+}\right)^{n}\right] \Phi_{\Lambda}\right)}{\left\|\left(O_{\Lambda}^{+}\right)^{n} \Phi_{\Lambda}\right\|^{2}}+\frac{\left(\Phi_{\Lambda},\left(O_{\Lambda}^{+}\right)^{n}\left[H_{\Lambda},\left(O_{\Lambda}^{-}\right)^{n}\right] \Phi_{\Lambda}\right)}{\left\|\left(O_{\Lambda}^{-}\right)^{n} \Phi_{\Lambda}\right\|^{2}}\right\} \\
& \leq \frac{1}{2 k+1} \sum_{n=1}^{k}\left\{\frac{2 n h(o N)^{2 n}}{b_{n}\left(\sigma_{\Lambda} N\right)^{2 n}-8 n^{2}(o N)^{2 n} N^{-1}}\right\} \\
& \leq 2 h k\left(\frac{o}{\sigma_{\Lambda}}\right)^{2 k}+O\left(N^{-1}\right),
\end{aligned}
$$

where we have made use of the estimates (7.18), (7.15) and assumption ii). 
Finally we calculate the (staggered) magnetization of the state $\Psi_{\Lambda}$,

$$
\begin{aligned}
& \left(\Psi_{\Lambda}, O_{\Lambda}^{(1)} \Psi_{\Lambda}\right)=\left(\Psi_{\Lambda}, O_{\Lambda}^{+} \Psi_{\Lambda}\right) \\
& =\frac{1}{2 k+1} \sum_{n=1}^{k}\left\{\frac{\left(\Phi_{\Lambda},\left(O_{\Lambda}^{-}\right)^{n}\left(O_{\Lambda}^{+}\right)^{n} \Phi_{\Lambda}\right)}{\left\|\left(O_{\Lambda}^{+}\right)^{n} \Phi_{\Lambda}\right\|\left\|\left(O_{\Lambda}^{+}\right)^{n-1} \Phi_{\Lambda}\right\|}+\frac{\left(\Phi_{\Lambda},\left(O_{\Lambda}^{+}\right)^{n}\left(O_{\Lambda}^{-}\right)^{n} \Phi_{\Lambda}\right)}{\left\|\left(O_{\Lambda}^{-}\right)^{n} \Phi_{\Lambda}\right\|\left\|\left(O_{\Lambda}^{-}\right)^{n-1} \Phi_{\Lambda}\right\|}\right\} \\
& =\frac{1}{2 k+1} \sum_{n=1}^{k}\left\{\sqrt{\frac{\left(\Phi_{\Lambda},\left(O_{\Lambda}^{-}\right)^{n}\left(O_{\Lambda}^{+}\right)^{n} \Phi_{\Lambda}\right)}{\left(\Phi_{\Lambda},\left(O_{\Lambda}^{-}\right)^{n-1}\left(O_{\Lambda}^{+}\right)^{n-1} \Phi_{\Lambda}\right)}}+\sqrt{\frac{\left(\Phi_{\Lambda},\left(O_{\Lambda}^{+}\right)^{n}\left(O_{\Lambda}^{-}\right)^{n} \Phi_{\Lambda}\right)}{\left(\Phi_{\Lambda},\left(O_{\Lambda}^{+}\right)^{n-1}\left(O_{\Lambda}^{-}\right)^{n-1} \Phi_{\Lambda}\right)}}\right\} \\
& \geq \frac{2}{2 k+1} \sum_{n=1}^{k} \sqrt{\frac{b_{n}\left(\Phi_{\Lambda},\left(O_{\Lambda}^{(1)}\right)^{2 n} \Phi_{\Lambda}\right)-8 n^{2}(o N)^{2 n} N^{-1}}{b_{n-1}\left(\Phi_{\Lambda},\left(O_{\Lambda}^{(1)}\right)^{2 n-2} \Phi_{\Lambda}\right)+8(n-1)^{2}(o N)^{2 n-2} N^{-1}}} \\
& \geq \frac{2}{2 k+1} \sum_{n=1}^{k} \sqrt{\frac{b_{n}\left(\Phi_{\Lambda},\left(O_{\Lambda}^{(1)}\right)^{2 n} \Phi_{\Lambda}\right)}{b_{n-1}\left(\Phi_{\Lambda},\left(O_{\Lambda}^{(1)}\right)^{2 n-2} \Phi_{\Lambda}\right)}}\left(1+O\left(N^{-1}\right)\right) \\
& \geq \frac{2 k}{2 k+1}\left\{\prod_{n=1}^{k} \frac{b_{n}\left(\Phi_{\Lambda},\left(O_{\Lambda}^{(1)}\right)^{2 n} \Phi_{\Lambda}\right)}{b_{n-1}\left(\Phi_{\Lambda},\left(O_{\Lambda}^{(1)}\right)^{2 n-2} \Phi_{\Lambda}\right)}\left(1+O\left(N^{-1}\right)\right)\right\}^{\frac{1}{2 k}} \\
& \geq \frac{2 k}{2 k+1}\left\{b_{k}\left(\Phi_{\Lambda},\left(O_{\Lambda}^{(1)}\right)^{2 k} \Phi_{\Lambda}\right)\right\}^{\frac{1}{2 k}}\left(1+O\left(N^{-1}\right)\right),
\end{aligned}
$$

where we bounded the arithmetic mean from below by the geometric mean. We also used the inequalities (7.15), (7.18), and the fact that $\lim _{N \uparrow \infty} \sigma_{\Lambda}=\sigma>0$. By using the lower bound in (7.19), we finally get

$$
\frac{1}{N}\left(\Psi_{\Lambda}, O_{\Lambda}^{(1)} \Psi_{\Lambda}\right) \geq \frac{2 k}{2 k+1} \times \frac{1}{N}\left(\Phi_{\Lambda},\left(O_{\Lambda}^{(1)}\right)^{2 k} \Phi_{\Lambda}\right)^{\frac{1}{2 k}}+O\left(N^{-1}\right)
$$

By substituting (7.24) and (7.26) into the basic variational estimate (7.5), and by letting $N \uparrow \infty$ and $k \uparrow \infty$, one gets the desired (7.11).

Remark. The estimate (7.24) of the energy expectation value of the variational state $\Psi_{\Lambda}$ only shows that the energy difference is smaller than a constant. This estimate is far from being optimal. In [16], we prove a much more refined version of the estimate (7.24), and discuss its relevance to the problem of low lying excited states in quantum systems in a finite volume.

Acknowledgements. We wish to thank Kenn Kubo, Elliott Lieb and Bruno Nachtergaele for useful discussion on related topics, and Bálint Tóth for useful remarks on the manuscript. The present research is supported in part by a Grant-in-Aid for General Scientific Research from the Ministry of Education, Science and Culture. 


\section{References}

1. Peierls, R.: Proc. Camb. Phil. Soc. 32, 447-481 (1936);

Griffiths, R.B.: Phys. Rev. A 136, 437-439 (1964);

Dobrushin, R.L.: Teorija Verojatn. i ee Prim. 10, 209-230 (1965) [Soviet Phys. Dokl. 10, 111 113 (1965)];

Robinson, D.: Commun. Math. Phys. 14, 195-204 (1969);

Ginibre, J.: Commun. Math. Phys. 14, 205-234 (1969);

Fröhlich, J., Lieb, E.H.: Commun. Math. Phys. 60, 233-267 (1978);

Kennedy, T.: Commun. Math. Phys. 100, 447-462 (1985)

2. Fröhlich, J., Simon, B., Spencer, T.: Commun. Math. Phys. 50, 79-95 (1976)

3. Glimm, J., Jaffe, A.: Quantum physics - a functional integral point of view. Berlin, Heidelberg, New York: Springer, 1981;

Fröhlich, J., Israel, R., Simon, B., Lieb, E.H.: Commun. Math. Phys. 62, 1-34 (1978) and J. Stat. Phys. 22, 297-347 (1980)

4. Dyson, F.J., Lieb, E.H., Simon, B.: J. Stat. Phys. 18, 335-383 (1978)

5. Kennedy, T., Lieb, E.H., Shastry, B.S.: J. Stat. Phys. 53, 1019-1030 (1988)

6. Kubo, K., Kishi, T.: Phys. Rev. Lett. 61, 2585-2587 (1988)

7. Jordão Neves, E., Fernando Perez, J.: Phys. Lett. 114 A, 331-333 (1986)

8. Kubo, K.: Phys. Rev. Lett. 61, 110-112 (1988);

Kennedy, T., Lieb, E.H., Shastry, B.S.: Phys. Rev. Lett. 61, 2582-2584 (1988);

Nishimori, H., Kubo, K., Ozeki, Y., Tomita, Y., Kishi, T.: J. Stat. Phys. 55, 259-277 (1989);

Ozeki, Y., Nishimori, H., Tomita, Y.: J. Phys. Soc. Jpn. 58, 82-90 (1989);

Nishimori, H., Ozeki, Y.: J. Phys. Soc. Jpn. 58, 1027-1030 (1989)

9. Kishi, T., Kubo, K.: J. Phys. Soc. Jpn. 58, 2547-2552 (1989);

Kishi, T., Kubo, K.: Phys. Rev. B 43, 10844-10852 (1991);

Saika, Y., Nishimori, H.: J. Phys. Soc. Jpn. 61, 3086-3095 (1992)

10. Griffiths, R.B.: Phys. Rev. 152, 240-246 (1966)

11. Kaplan, T.A., Horsch, P., von der Linden, W.: J. Phys. Soc. Jpn. 58, 3894-3897 (1989)

12. Bratteli, O., Robinson, D.W.: Operator algebras and quantum statistical mechanics I, II. Berlin, Heidelberg, New York: Springer, 1979

13. Van Hove, L.: Physica 15, 951-961 (1949)

14. Griffiths, R.B.: J. Math. Phys. 5, 1215-1222 (1964);

Ruelle, D.: Statistical mechanics: rigorous results. Reading, Massachusetts: W.A. Benjamin, 1969

15. Horsch, P., von der Linden, W.: Z. Phys. B 72, 181-193 (1988)

16. Koma, T., Tasaki, H.: Obscured symmetry breaking and low lying excited states. Preprint 